\title{
Archive in Frankreich
}

Florence de Peyronnet-Dryden et Matthias Nuding

\section{OpenEdition}

\section{Journals}

Édition électronique

URL : http://journals.openedition.org/ifha/7877

DOI : 10.4000/ifha.7877

ISSN : 2198-8943

\section{Éditeur}

IFRA - Institut franco-allemand (sciences historiques et sociales)

\section{Référence électronique}

Florence de Peyronnet-Dryden und Matthias Nuding, "Archive in Frankreich », Revue de I'IFHA [Online], HS | 2014, Online erschienen am: 01 September 2014, aufgerufen am 30 April 2019. URL : http:// journals.openedition.org/ifha/7877 ; DOI : 10.4000/ifha.7877

Ce document a été généré automatiquement le 30 avril 2019

(CIFHA 


\title{
Archive in Frankreich
}

\author{
Florence de Peyronnet-Dryden et Matthias Nuding
}

\section{Vom Dokument zum Archiv}

1 Archivalien sind Dokumente, die im Zuge der Tätigkeit einer Person oder einer Behörde produziert oder von ihr empfangen wurden und diese Tätigkeit dokumentieren, unabhängig von der Form, Beschaffenheit, Materialität und Entstehungszeit der Aufzeichnungen ${ }^{1}$. Sie dienen nicht per se der historischen Forschung. Nachdem man sie in den meisten Fällen aufgrund ihrer Beweiskraft aufbewahrte, wurden sie erst im Laufe der Zeit zu geschichtlichen Quellen.

2 Der französische Sprachgebrauch weicht in mehrfacher Hinsicht vom deutschen ab, was $\mathrm{zu}$ Missverständnissen führen kann. Es ist wichtig, sich diese Unterschiede zu vergegenwärtigen: Zum einen differenziert das Französische nicht zwischen den Begriffen »Archiv« und »Archivalien« (»Archivgut«). Alles heißt les archives (im Plural), seien es die betreffenden Dokumente, die Archivbehörden oder die Gebäude, in denen die Archivalien aufbewahrt werden.

Zum anderen werden Unterlagen schon als archives bezeichnet, bevor sie in ein Archiv übernommen werden. Die französische Archivwissenschaft unterscheidet drei Kategorien von archives (théorie des trois âges): Solange die Aufzeichnungen von der Person oder Stelle, bei der sie entstanden sind, regelmäßig benutzt werden, nennt man sie archives courantes (Registraturgut). Behält der Besitzer sie nach dieser Phase aus verwaltungstechnischen oder juristischen Gründen noch für eine gewisse Frist in seiner Obhut, werden sie zu archives intermédiaires (Altregistratur). In einer dritten und letzten Phase können sie zu archives définitives (Archivgut) umgewidmet und in ein Archiv übernommen werden. Das betrifft solche Unterlagen, denen man eine so große Bedeutung beimisst, dass man sie als dauerhaft aufbewahrungswürdig bewertet. Nur diese archives définitives sind Archivgut im Sinne der deutschen Sprache; sie findet man vor, wenn man ein Archiv besucht.

4 Juristisch unterscheidet man in Frankreich zwischen öffentlichen (staatlichen) und privaten Archiven. Die Letzteren - z. B. Familien-, Verbands-, Unternehmens-, Kirchen- 
und Parteienarchive - unterliegen nicht den Regelungen, die für öffentliche Archive gelten. In der Praxis sind die Grenzen dieser Einteilung bisweilen durchlässig: So befinden sich viele Bestände nicht öffentlicher Provenienz noch ganz in Privatbesitz, andere liegen zwar in öffentlichen Institutionen, oft aber nur als Deposita (d. h. hinterlegt ohne Eigentumsübertragung). Archive wichtiger Adelsfamilien, die auch eine bedeutende Rolle in der Geschichte Frankreichs im Ancien Régime spielten, wurden während der Revolution zusammen mit den Gütern dieser Familien (biens nationaux) konfisziert und befinden sich daher in den Archives nationales oder in den Archives départementales. In diesen öffentlichen Archiven werden also nicht nur Unterlagen aus der Zentral- bzw. Lokalverwaltung aufbewahrt. Man findet dort auch Bestände, die ursprünglich privater oder halböffentlicher Natur waren (Nachlässe, Adelsarchive, Archive von Hospitälern, Kirchen etc.) und die aus verschiedenen Gründen dorthin gelangten.

\section{Ordnung von Archiven}

5 Um die langfristige Archivierung zu sichern, müssen die Dokumente nicht nur fachgerecht konserviert, sondern auch entsprechend identifiziert bzw. inventarisiert und geordnet werden. Die Grundlage dafür ist das so genannte Provenienzprinzip (principe de respect des fonds). Es besagt, dass Archivalien nicht willkürlich nach Themengebieten in künstlichen Sammlungen geordnet werden, sondern möglichst die Herkunfts- und Entstehungszusammenhänge erhalten bleiben. Somit werden zusammengehörende Archivalien, die einen Bestand (fonds) bilden, nicht voneinander getrennt ${ }^{2}$.

Diese Bestände werden in Archiven der öffentlichen Hand entsprechend einem nach Perioden und Themen gegliederten Ordnungsschema (cadre de classement) abgelegt. Im Gegensatz zu den Zuständen in deutschen Archiven gilt dieses Schema einheitlich für alle staatlichen Archive in Frankreich. Jede seiner Ebenen heißt série. Zu jeder série gehören wiederum verschiedene Bestände, die als sous-séries bezeichnet werden ${ }^{3}$. Auf der Ebene eines jeden Bestandes findet man den plan de classement, der ebenfalls jede Archivaliensammlung nach Themen, Dokumentenarten oder/und Perioden inhaltlich strukturiert.

7 Die séries sind mit Buchstaben gekennzeichnet, die für alle Archives départementales die gleichen Bereiche abdecken: In »D« findet man die »Instruction publique, sciences et arts« (Bildung, Wissenschaft und Kunst), in »H« den »Clergé régulier« (Ordensklerus). Die gleichen Buchstaben haben jedoch eine andere Bedeutung in den Archives nationales: "D« sind die »Missions des représentants du peuple et comités des assemblées" (Delegationen der Vertreter des Volkes [= Zeit der französischen Revolution]), "H« dagegen » Administrations locales et collectivités diverses" (Lokalverwaltungen [in der Zeit des Ancien Régime]). In den Archives nationales findet man sogar Doppelbuchstaben (»K« und »KK« für ausgewählte historische Dokumente, oder »AD« für Drucksachen), Kommunalarchive haben für die Zeit vor 1790 Doppelbuchstaben (»CC« für Finanzen).

\section{Aufgaben von Archivarinnen und Archivaren}

Die archivarische Tätigkeit ist vielfältig und kann in vier Hauptbereiche unterteilt werden: die Sammlung von Archivalien, die Pflege des Archivgutes, die Erschließung der Bestände und deren Vermittlung an die Öffentlichkeit. Das heißt, von der Übernahme 
archivwürdigen Materials und der Organisation seiner fachgerechten Konservierung und Restaurierung reicht die Tätigkeit über die Ordnung und Verzeichnung der Bestände (die Kernaufgabe des Archivars) bis hin zur Organisation von Ausstellungen und Kolloquien sowie nicht zuletzt zur Beratung der Benutzer. Unter Beratung versteht man die Orientierung der Leserschaft in ihren Forschungsvorhaben: Archivare beantworten Fragen zur Suche nach Quellen, geben Tipps zu den für die Thematik interessanten Beständen, erklären die Beständestruktur und können auch die Reproduktion von Dokumenten veranlassen, wenn Umfang des Auftrags und Rechte bzw. Zustand der Archivalien dies erlauben. Ihre Aufgabe ist es aber nicht, Texte zu transkribieren oder selber in den Beständen zu forschen (außer für kurze Überprüfungen), um die Fragen der Forschenden zu beantworten; genauso wenig könnte man von Bibliothekaren erwarten, dass sie ein Buch für einen Leser lesen und ihm die Zusammenfassung schicken ... Das ist nicht zuletzt aus Kapazitätsgründen unmöglich.

\section{Die Ausbildung der französischen Archivarinnen und Archivare}

Die wissenschaftlichen Archivare des höheren Dienstes werden in Frankreich seit 1821 in der École des Chartes ausgebildet ${ }^{4}$. Nach Vorbereitungsklassen (in der Regel zwei bis drei Jahre), gefolgt von einer Aufnahmeprüfung (concours), werden sie in diese grande école aufgenommen. Im Anschluss an eine dreijährige theoretische Ausbildung und die Vorlage einer thèse müssen sie noch knapp zwei Jahre den praktischen Teil (eine Art Referendariat) im Institut national du Patrimoine absolvieren. Nach dieser langen und anspruchsvollen Ausbildung werden sie zu Conservateurs du Patrimoine ernannt und können somit ihre Verwaltungslaufbahn beginnen. Seit circa 25 Jahren gibt es auch die Möglichkeit, über spezielle Universitätslehrgänge Archivar zu werden. Ein Teil dieser Absolventen wird nach einer Aufnahmeprüfung in das Institut national du Patrimoine (für den höheren Dienst) oder direkt in die Archivverwaltung (für den gehobenen Dienst) aufgenommen, andere wiederum wenden sich zum Beispiel an Unternehmensarchive ${ }^{5}$.

\section{Gründung des Nationalarchivs und Archivlandschaft}

10 Die heutige französische Archivorganisation ist im Zuge der Französischen Revolution entstanden. Die neue Regierung wollte einen spezifischen Verwaltungsapparat schaffen, der drei Hauptaufgaben übernehmen sollte: die Aufbewahrung der von den neuen Behörden produzierten Dokumente, das Sammeln der Unterlagen aus der Zeit des Ancien Régime und die Übernahme der konfiszierten Bestände aus den sogenannten Nationalgütern. Trotz aller Vernichtungen von Kulturgut, zu denen es in ihrem Verlauf gekommen ist, hat die Revolution damit auch die Rettung und Bewahrung historischer Überlieferung ermöglicht.

11 Das französische Nationalarchiv (Archives nationales) wurde per Dekret vom 7. September 1790 gegründet, dem das Gründungsgesetz vom 7. Messidor II (25. Juni 1794) folgte ${ }^{6}$. Drei neue Grundgedanken wurden in diesem Gesetz definiert:

- die archives der Nation sollten künftig zentral aufbewahrt werden;

- dieses Archivgut war nun öffentlich - im Gegensatz zum Ancien Régime, das sich auf das Prinzip des Staatsgeheimnisses berufen hatte; 
- diese Gründung sollte zudem mit dem Aufbau eines nationalen Archivnetzwerks verbunden werden?

Ein weiteres Gesetz rief die Archivbehörden für diejenigen Bestände ins Leben, die in der Provinz aufbewahrt wurden; damit bekam jedes Département ein eigenes Archiv ${ }^{8}$. Das 19. Jahrhundert setzte dieses System fort, ohne an den Weichenstellungen von $1790 \mathrm{zu}$ rütteln; die Grundstrukturen sind bis heute weitgehend unverändert geblieben.

\section{Organisation der öffentlichen Archive}

13 Charakteristisch für die französische Archivlandschaft ist daher ihre - abgesehen von einigen Ausnahmen - sehr einheitliche Organisation. Diese ist eine positive Folge ihrer geschichtlichen Entwicklung, des zentralisierenden jakobinischen Gedankens der Revolution. Auch nach den dezentralisierenden Maßnahmen der jüngsten Jahrzehnte bleibt das Gesamtbild homogen und verständlich: Anders als in Deutschland ${ }^{9}$ können Forschende sich relativ schnell und autonom mit der Archiv- und Beständestruktur vertraut machen.

Zusammenfassend kann man die öffentlichen Archive in drei Gruppen unterteilen:

- Das Nationalarchiv verwahrt die Archivbestände der Zentralbehörden des französischen Staates. Ursprünglich in Paris angesiedelt - seit Napoleon I. im Palais Soubise, wo ein Teil der Bestände sich heutzutage immer noch befindet -, sind die Archives nationales seit Anfang des Jahres 2013 auf drei Standorte verteilt: auf Paris, Fontainebleau (seit 1969) und den am 21. Januar 2013 eröffneten Neubau von Pierrefitte-sur-Seine, nördlich von Paris. Während man in Paris die Bestände bis zur Französischen Revolution sowie die Archive der Pariser Notare (»Minutier central«) aufbewahrt, liegt die Überlieferung ab der Revolution je nach Themengebiet entweder in Fontainebleau oder in Pierrefitte ${ }^{10}$. Wegen des rasanten Wachstums der Bestände einerseits und aus dem Wunsch heraus, die Archive zu dezentralisieren, andererseits haben einige Abteilungen einen eigenen Standort erhalten: Seit 1969 besteht in Aix-en-Provence das Centre des archives d'outre-mer (Archiv der Überseeterritorien, das die Unterlagen der ehemaligen Kolonialverwaltung aufbewahrt) ${ }^{11}$, die Archives nationales du monde du travail (Archiv der Arbeitswelt, mit Unterlagen zu Unternehmen, Gewerkschaften, Verbänden und Architekten) ${ }^{12}$ befinden sich seit 1993 in Roubaix, und das Centre national du microfilm et de la numérisation (Mikrofilm- und Digitalarchiv) ${ }^{13}$ hat seit 1973 seinen Sitz in Saint-Gilles-du-Gard.

- Drei Archive von Zentralbehörden wurden nicht in das Nationalarchiv integriert: Es handelt sich zum einen um das Archiv des Verteidigungsministeriums (Service historique de la Défense) $)^{14}$, das sich in Vincennes bei Paris befindet, dann um das Archiv des Außenministeriums (Archives du Ministère des Affaires étrangères) ${ }^{15}$, früher in Paris, Quai d'Orsay, nun in einem Neubau nördlich von Paris in La Courneuve, und schließlich um das Archiv des Wirtschafts- und Finanzministeriums (Centre des archives économiques et financières $)^{16}$ in Savigny-le-Temple, unweit von Paris. Diese Archive hatten schon im Ancien Régime eine ausgeprägte Eigenständigkeit und wurden wegen ihrer zentralen Bedeutung für den Staat unter den gleichen Vorzeichen weitergeführt.

- Die wichtigsten Archive der Provinz sind die in jedem Département einheitlich strukturierten Archives départementales ${ }^{17}$. Größere Gemeinden besitzen ebenfalls eigene Archive (Archives municipales), wohingegen die Archive kleinerer Gemeinden in den meisten Fällen im Archiv des Départements deponiert werden ${ }^{18}$. Die nach dem Zweiten Weltkrieg aus jeweils mehreren Départements errichteten Regionen haben seit den 1980er 
Jahren zudem eigene Archives des Régions, die allerdings nur die jüngste Geschichte überliefern.

Die Kontrolle der öffentlichen Archive sowie der Schutz privater Archive in Frankreich obliegt dem Service interministériel des archives ${ }^{19}$. Er untersteht dem Ministère de la Culture und veröffentlicht jedes Jahr ein Adressbuch über die wichtigsten Archive in Frankreich $^{20}$, die wir hier zusammenfassend vorstellen.

\section{Archive anderer Träger}

Auch andere Institutionen unterhalten Archive, die historisch Arbeitende bei ihren Recherchen berücksichtigen sollten. Die folgende Liste enthält einige Beispiele:

- Krankenhäuser archivieren ihre eigenen Unterlagen meist selbst (Archives hospitalières) ${ }^{21}$; in manchen Fällen werden sie jedoch an öffentliche Archive abgegeben.

- Verschiedene kulturelle Institutionen wie Bibliotheken ${ }^{22}$ oder Museen sind im Besitz von Nachlässen, die man als Archivbestände bezeichnen kann und die oft große Schätze für die Geschichtsforschung enthalten. Als Beispiele seien hier die Bibliothek des Institut de France in Paris ${ }^{23}$ oder der umfangreiche Bestand des Muséum d'histoire naturelle ${ }^{24}$ erwähnt.

- Verbände, Gewerkschaften usw. haben ebenfalls eigene Archive; zum Teil wurden ihre Bestände aber auch an die Archives nationales oder Archives départementales abgegeben.

- Die katholische Kirche unterhält Archive in den Diözesen, Kongregationen und sonstigen Einrichtungen, die protestantischen Kirchen bei den jeweiligen Gemeinden. Allerdings wurden die Unterlagen bis zur Französischen Revolution (bzw. 1801) laut Gesetz vom 5. Januar 1790 an den Staat übergeben ${ }^{25}$. Ähnliche Verhältnisse findet man bei den jüdischen Gemeinden ${ }^{26}$.

- Auch Unternehmen haben eigene Archive, die für die Geschichte der jeweiligen Firma und mithin für die Wirtschafts- und Finanzgeschichte von Bedeutung sind ${ }^{27}$.

- Medien- und Bildarchive findet man hauptsächlich bei zwei Organisationen: den Archives françaises $d u$ film ${ }^{28}$ des Centre national du cinéma et de l'image animée und dem Institut national de l'Audiovisuel (INA) ${ }^{29}$.

- Privatpersonen oder Familien können ebenfalls Archive unterhalten. Je nach ihrer Rolle und Wichtigkeit können darin wertvolle Informationen zu bestimmten Personen, aber auch zur Geschichte der Region oder des Landes enthalten sein.

\section{Benutzung der Archive}

Bis zur Verabschiedung einschlägiger Gesetze war der Zugang zu den Archiven nicht einheitlich geregelt und wurde - auch in Frankreich - eher restriktiv gehandhabt. Die vor wenigen Jahrzehnten entwickelte Archivgesetzgebung hat einen Ausgleich zwischen den gegensätzlichen Interessen des Datenschutzes und des öffentlichen Informationsbedürfnisses herbeizuführen vermocht und damit eine Rechtssicherheit geschaffen, die den Archivnutzern zugutekommt.

Die meisten öffentlichen Archive in Frankreich stehen heute jedem Interessierten offen, der sich ausweisen kann. Seit 2004/08 richtet sich die Benutzung der Staats-, Regional-, Départemental- und Kommunalarchive nach dem zweiten Buch des Code du Patrimoine ${ }^{30}$, welches das Archivgesetz von 1979 abgelöst hat ${ }^{31}$. Darin ist u.a. festgelegt, dass grundsätzlich fast alle in diesen Archiven verwahrten Unterlagen zur Benutzung 
freigegeben sind, sofern dem keine speziellen Schutz- und Sperrfristen entgegenstehen ${ }^{32}$. Solche Fristen gelten indessen nach Artikel L. 213-2 für einige Kategorien von Archivgut besonders sensiblen Inhalts, nämlich:

- 25 Jahre für Unterlagen über Beratungen der Regierung und anderer Organe der vollziehenden Gewalt, über die auswärtigen Beziehungen, das Geldwesen, die öffentlichen Finanzen, Handels- und Wirtschaftsgeheimnisse, Steuer- und Zollvergehen, vertrauliche Statistiken, außerdem für personenbezogene Dokumente, deren Inhalt unter die ärztliche Schweigepflicht fällt (wobei die Freigabe 25 Jahre nach dem Tod des Betroffenen, hilfsweise 120 Jahre nach der Geburt erfolgt).

- 50 Jahre für Unterlagen $\mathrm{zu}$ Fragen der Landesverteidigung, $\mathrm{zu}$ grundlegenden außenpolitischen Interessen des Staates, zur Sicherheit von Staat und Bürgern, zum Schutz der Privatsphäre, ferner Dokumente mit Beurteilungen identifizierbarer Personen oder Unterlagen über Einrichtungen des Strafvollzugs (gerechnet ab dem Ende ihrer Nutzung als solche).

- 75 Jahre (oder 25 Jahre nach dem Tod des Betroffenen, falls diese Frist kürzer ist) für Dokumente vertraulichen statistischen Inhalts und solche über polizeiliche Ermittlungen, für bestimmte Unterlagen der Rechtsprechung und öffentlicher Amtsträger sowie für standesamtliche Geburts- und Heiratsregister.

- 100 Jahre (oder 25 Jahre nach dem Tod des Betroffenen, falls diese Frist kürzer ist) für Unterlagen mit einer Sperrfrist von 75 Jahren, falls der Betroffene noch minderjährig war, für geheime Unterlagen der Landesverteidigung, deren offenlegung die Sicherheit identifizierbarer Personen gefährden könnte, sowie für Akten von Polizei und Justiz, sofern sie Aufschluss über das Geschlechtsleben Einzelner geben.

19 Die nicht durch ein Geburts- oder Todesdatum terminierten Fristen beginnen mit dem Datum des Dokuments bzw. des jüngsten Dokuments, das in der jeweiligen Archivguteinheit enthalten ist. Auf Antrag kann in begründeten Fällen eine Verkürzung dieser Fristen (dérogation) unter Auflagen gewährt werden. Der Antrag ist an das verwahrende Archiv zu richten, das ihn zur Entscheidung an den Service interministériel des Archives de France weiterleitet. Die Erfolgsaussichten solcher Anträge sind nach den bisherigen Erfahrungen relativ gut ${ }^{33}$.

20 Für Archivgut privater Herkunft (etwa Nachlässe) können andere, individuell vereinbarte Zugangsfristen gelten. Unterhalb der gesetzlichen Ebene gibt es von Archiv zu Archiv auch eigene Benutzungs- oder Lesesaalordnungen.

\section{Wie finde ich das richtige Archiv?}

21 Am Beginn jeder Archivrecherche steht zunächst die Suche nach dem richtigen Archiv, das Quellen über den zu untersuchenden Gegenstand bereithält ${ }^{34}$. Da Archivalien in der Regel Unikate sind, findet man sie meist auch nur an einem Ort überliefert. Je nach der Komplexität der Fragestellung kann das einschlägige Material sich selbstverständlich auf mehrere Archive verteilen. Das liegt dann allerdings in der Regel nicht daran, dass Teile desselben Bestandes in verschiedene Archive gelangt wären, sondern daran, dass mehr als ein Bestand relevantes Material enthält. Sofern vorhanden, können allgemeine Findmittel wie Übersichten über die Bestände einzelner Archive (états des fonds) oder zu bestimmten Themen (guides d'archives) den Weg weisen ${ }^{35}$. 
22 Der archivalischen Überlieferung liegt in den meisten Fällen eine verwaltungsgeschichtliche Logik zugrunde. Die Unterlagen einer öffentlichen Stelle werden, wenn sie im Dienstbetrieb entbehrlich geworden sind, vorschriftsgemäß dem für diese Stelle zuständigen Archiv angeboten, d.h. die Überlieferung einer staatlichen Behörde findet sich im jeweiligen staatlichen Archiv, die Überlieferung einer Kommune im Kommunalarchiv usw. Im Falle nicht mehr existenter Registraturbildner (etwa historischer Institutionen) wird man gewöhnlich im Archiv des jeweiligen Rechts- oder Funktionsnachfolgers fündig. Dabei muss man freilich im Auge behalten, dass - wie oben bereits angedeutet - manche Gegebenheiten in der französischen Archivlandschaft aus historischen Gründen eigenen Gesetzen folgen. Insbesondere bei Archivgut privater Herkunft ${ }^{36}$ verläuft die Überlieferungsbildung oft außerhalb dieser standardisierten Bahnen, so dass Informationen über die Existenz oder den Verbleib solcher Dokumente im Einzelfall etwas schwieriger zu erlangen sein können.

Eine wichtige Orientierungshilfe stellt das bereits genannte, nach Sparten gegliederte Adressenverzeichnis dar, das auf der Internetseite des Service interministériel des Archives de France konsultiert werden kann $^{37}$. Daneben gibt es im Internet weitere einschlägige Verzeichnisse ${ }^{38}$.

\section{Kontaktaufnahme mit einem Archiv}

Hat man das richtige Archiv identifiziert, kann man die Suche schon vor dessen Besuch verfeinern, soweit es die im Druck oder online veröffentlichten Findmittel (siehe unten) erlauben. Sind solche nicht oder nicht in hinreichendem Maße vorhanden, empfiehlt sich eine direkte Kontaktaufnahme mit dem Archiv (die bei komplexeren Anliegen vor einem Archivbesuch ohnehin empfehlenswert ist). Diese Anfrage sollte auf schriftlichem Wege erfolgen und das Benutzungsanliegen so ausführlich wie nötig darstellen, denn fundierte und erschöpfende Auskünfte erhält man nicht unbedingt aus dem Stegreif am Telefon ${ }^{39}$. Zusätzlich zu den öffentlich verfügbaren Informationsquellen kommt dem individuellen Kontakt mit dem zuständigen Archivar nach wie vor eine hohe Bedeutung zu. Eventuell kann dieser offene Fragen beantworten oder Hinweise auf weitere einschlägige Unterlagen innerhalb oder außerhalb seines Hauses geben.

\section{Archivfindmittel im Wandel der Zeit}

Die meisten Archive haben erst deutlich später als die Bibliotheken damit begonnen, ihre Bestandskataloge digital zu führen und im Internet zur Verfügung zu stellen. Seitdem jedoch die technischen und konzeptionellen Grundlagen vorhanden sind, entwickelt sich diese Art der Erschließung auch im Archivwesen mehr und mehr zum Standardfall. Die Abfassung archivischer Findmittel orientiert sich dabei zunehmend an dem international einheitlichen XML-Dokumentationsstandard EAD (Encoded Archival Description), der die strukturierte Darstellung und Auswertung der Daten (auch in übergreifenden elektronischen Katalogen) erlaubt.

Archivische Online-Ressourcen sind in den weitaus meisten Fällen freilich nur beschreibende Metadaten (Angaben über Inhalt, Laufzeit u.a.), keine Volltexte. Wegen des hohen Aufwands, den eine entsprechende Aufbereitung erfordert, stehen allenfalls einzelne, besonders wichtige oder viel nachgefragte Archivbestände vom heimischen 
Rechner aus zur Verfügung. Die meisten Unterlagen kann man weiterhin nur vor Ort im Archiv sichten. Von dieser Regel gibt es inzwischen allerdings mehr und mehr Ausnahmen (siehe unten im Abschnitt »Quellen und Archivalien digital«).

Zur Ermittlung der relevanten Unterlagen stehen im Archiv verschiedene Hilfsmittel ( répertoires, inventaires) zur Verfügung: Die klassischen Formate sind papierene Findbücher oder Karteien (nicht selten noch handschriftlich); im Gegensatz zu den digitalen Katalogen bieten sie keine dynamischen Recherchefunktionen und stehen auch nicht außerhalb des Archivs zur Verfügung. Beide Generationen archivischer Findmittel unterscheiden sich in verschiedener Hinsicht von Bibliothekskatalogen. So sind die darin enthaltenen Beschreibungen häufig keine vorgegebenen Titel, sondern vom Archivar ermittelte Angaben, die mehr oder weniger erschöpfend ausfallen; des besseren Verständnisses wegen werden Archivalien außerdem nicht als gleichrangige Einzeldokumente, sondern eingebettet in ihren hierarchisch gegliederten Überlieferungszusammenhang dargestellt. Über Geschichte, Aufbau und Inhalt des jeweiligen Bestandes informiert dabei die Einleitung des Findbuchs. Jede archivalische Verzeichnungseinheit (z.B. Urkunde oder Akte) trägt eine eindeutige Signatur (cote), anhand derer man sie zur Einsichtnahme bestellen kann ${ }^{40}$.

Während das Archivpersonal bei der Orientierung im Archiv, beim Umgang mit den Repertorien und bei der Aufgabe von Bestellungen im Bedarfsfall behilflich ist, bleibt der Leser beim Studium der Archivalien auf sich allein gestellt. Je nach Region und Epoche muss man dabei mit historischen Schriften und Sprachformen rechnen, zu deren Verständnis einschlägige Kenntnisse unerlässlich sind ${ }^{41}$.

Viele Archive bieten die Möglichkeit an, Reproduktionen (Scans, Fotos, Fotokopien o.ä.) aus ihren Beständen zu bestellen, manche erlauben sogar den Gebrauch mitgebrachter Digitalkameras ${ }^{42}$. Einzelheiten lassen sich oft bereits dem Informationsangebot des Archivs (z.B. auf der Internetseite) entnehmen. Grundsätzlich hat der Archivnutzer die gewünschten Dokumente selbst herauszusuchen. Benötigt man jedoch nur Reproduktionen einzelner Blätter, deren Signaturen man bereits kennt, kommt eventuell auch eine schriftliche Bestellung ohne persönlichen Archivbesuch in Frage. In solchen Fällen ist allerdings auf die genaue Bezeichnung der Dokumente und auf die Benutzung des eventuell vorgesehenen Bestellformulars zu achten.

\section{Quellen und Archivalien digital: Digitalisierungsprojekte}

In jüngerer Zeit werden immer mehr Bestände französischer Archive in Form von Digitalisaten über das Internet zur Verfügung gestellt. Auch wenn es sich nur um besonders bedeutende Unterlagen handelt, deren Umfang im Verhältnis zur Masse des übrigen Archivguts noch relativ gering ist, wächst der Umfang dieser collections numérisées stetig $\mathrm{an}^{43}$. Eine nützliche Übersicht über einschlägige Projekte und Online-Angebote bietet die vom Kulturministerium betriebene Internetseite Patrimoine numérique ${ }^{44}$, auf der auch eine Auswahl nach Institutionen (Archiven, Bibliotheken, Museen etc.) getroffen werden kann. Das Centre historique des Nationalarchivs macht mit der Datenbank ARCHIM, die derzeit in neunzehn thematische Rubriken gegliedert ist, digitalisierte Quellen aus seinen Beständen zugänglich ${ }^{45}$. Daneben gibt es auch bereits eine ganze Reihe von Spezialbeständen, die online einsehbar sind ${ }^{46}$. 


\section{Archive und die deutsch-französische Geschichte}

31 naturgemäß in den Archiven beiderseits der Grenze Spuren hinterlassen. Französische Archive - nicht nur solche in den Grenzregionen - verwahren eine ganze Reihe von Beständen, die für die deutsche Geschichte aussagekräftig sind ${ }^{47}$; in Einzelfällen handelt es sich sogar um Unterlagen, die in Deutschland oder bei deutschen Stellen entstanden sind - etwa was die militärischen oder zivilen Besatzungsverwaltungen im 20. Jahrhundert betrifft ${ }^{48}$. Solche Bestände wurden in der Vergangenheit wiederholt im Rahmen konzertierter deutsch-französischer Projekte erschlossen, an denen u.a. das in Paris ansässige Deutsche Historische Institut ${ }^{49}$ beteiligt war. Diese Einrichtung, deren Aufgabe darin besteht, als Drehscheibe zwischen deutschen und französischen Geschichtswissenschaftlern zu fungieren, kann bei einschlägigen Forschungsvorhaben ein interessanter Ansprechpartner sein - nicht zuletzt deshalb, weil sie neben fachlichem Rat auch Kurzzeitstipendien und Gästezimmer für Archivaufenthalte in Paris bereithält.

Das wohl aktuellste einschlägige Projekt ist das von der Europäischen Union mitfinanzierte grenzüberschreitende Portal für Archivgut am Oberrhein, das sich derzeit unter dem Arbeitstitel »Archivum Rhenanum« im Aufbau befindet ${ }^{50}$. Dieses Vorhaben, an dem zahlreiche französische und deutsche Archive aus der Region beteiligt sind, kann als Beispiel für den Trend zur Entgrenzung - sowohl im nationalen wie im medialen Sinne gesehen werden, der die Zukunft des Archivwesens prägen wird ${ }^{51}$.

\section{NOTES}

1. Definition aus dem französischen Archivgesetz vom 3. Januar 1979. Siehe: http:// www.legifrance.gouv.fr/ affichTexte.do;jsessionid=25D171A031567B2C09911403AEFAD388.tpdjo13v_3? cidTexte=JORFTEXT000000322519\&dateTexte $=19790105$. Alle Links wurden am 15.7.2014 überprüft. Zur Bedeutung der Archive für die Geschichtsforschung siehe außerdem: Nicolas Offenstadt, Archives, documents, sources, in: Christian Delacroix, François Dosse, Patrick Garcia, Nicolas Offenstadt (Hg.), Historiographies, Bd. 2, Paris 2010, S. 68-78; außerdem Arlette Farge, Le goût de l'archive, Paris 1989.

2. Vgl. dazu: Direction des Archives de France, La pratique archivistique française, Paris ${ }^{2} 2008$.

3. Vgl. dazu: Association des archivistes français (Hrsg.), Abrégé d'archivistique, Paris ${ }^{3} 2012$.

4. Informationen über die École des Chartes findet man unter http://www.enc.sorbonne.fr/.

5. Weitere Auskünfte über die Ausbildung und das Berufsbild gibt es bei der Association des archivistes français, dem Berufsverband der französischen Archivare: http:// www.archivistes.org/.

6. Zur Geschichte des Nationalarchivs und der Archivorganisation in Frankreich: Claire Béchu (dir.), Les Archives nationales. Des lieux pour l'histoire de France. Bicentenaire d'une installation, 1808-2008, Paris, 2008; Lucie Favier, La mémoire de l'État. Histoire des Archives nationales, Paris, 2004; Françoise Hildesheimer, Les Archives de France. Mémoire de l'Histoire, Paris, 1997. Über 
die Geschichte der Archives nationales siehe ebenfalls die Website dieser Institution über: http:// www.archives-nationales.culture.gouv.fr/sia/web/guest/histoire-de-l-institution.

7. D.h. Verbindungen und Zusammenarbeit mit den verschiedenen Ebenen der geplanten staatlichen Archive.

8. Geschichtliche Hintergründe wurden der folgenden Website entnommen: http:// www.archivesdefrance.culture.gouv.fr/archives-publiques/historique/. Weiterführende Informationen ebenfalls unter dieser Adresse.

9. Falk Bretschneider, Juliette Guilbaud, Les archives en Allemagne, in: Ders., Mareike König (Hg.), Faire de l'histoire en Allemagne: un guide pour les jeunes chercheurs français, Scholar Guide 1 (2011), http://www.perspectivia.net/content/publikationen/scholar-guide/histoire-enallemagne/bretschneider-guilbaud_archives.

10. Bis einschließlich 2012 beherbergte Fontainebleau nur die jüngsten zeitgenössischen Bestände, das heißt beginnend ab 1958.

11. Informationen über folgende Website: http://www.archivesnationales.culture.gouv.fr/anom/ $\mathrm{fr} /$.

12. Obwohl es sich hier nicht im eigentlichen Sinne um Bestände öffentlicher Behörden handelt, wurden sie in das Nationalarchiv integriert, da sie Überlieferungsgut von nationalem und öffentlichem Interesse bilden. Siehe dazu: http://www.archivesnationales.culture.gouv.fr/camt/. 13. Es enthält die originalen Mikroformen der in den anderen nationalen und regionalen Archiven verwahrten Dokumente. Hier wird auch die Digitalisierung der Dokumente vorgenommen.

14. Vgl. Service historique de la Défense, http://www.servicehistorique.sga.defense.gouv.fr/.

15. Vgl. Archives diplomatiques, http://www.diplomatie.gouv.fr/fr/photos-videos-etpublications/archives-et-patrimoine/.

16. Vgl. Centre des archives économiques et financières, http://www.economie.gouv.fr/caef/ accueil-caef.

17. Vgl. Archives départementales, http://fr.wikipedia.org/wiki/Archives_d\%C3\% A9partementales.

18. Laut dem Code du Patrimoine sind Gemeinden mit weniger als 2000 Einwohnern zudem verpflichtet, die Akten des Standesamts, die älter als 150 Jahre sind, in den Archives départementales zu deponieren; für Katasterunterlagen, die älter als 30 Jahre sind, gilt dieselbe Regel, sowie für alle anderen Dokumente, die älter als 100 Jahre sind.

19. So die neue Bezeichnung seit 2009; vorher hieß diese 1897 gegründete Einrichtung Direction des Archives de France.

20. Online-Version über: Annuaire des services d'archives, http:// www.archivesdefrance.culture.gouv.fr/annuaire-services/.

21. Vgl. Archives hospitalières, http://fr.geneawiki.com/index.php/Archives_hospitali\%C3\% A8res. Die Pariser Krankenhäuser und Spitäler der Assistance publique haben ein Portal zur Suche von Akten des 18. und 19. Jahrhunderts (bis 1870) eingerichtet: http://recherche.aphp.fr/. 22. Vgl. Abes, Catalogue en ligne des archives et des manuscrits de l'enseignement supérieur, http://www.calames.abes.fr/pub/.

23. Siehe deren Handschriftenbestände: http://www.institut-de-france.fr/fr/patrimoinemusees/biblioth\%C3\%A8que-de-linstitut.

24. Vgl. Muséum national d'histoire naturelle, direction des bibliothèques et de la documentation, http://bibliotheques.mnhn.fr/medias/medias.aspx?INSTANCE=EXPLOITATION.

25. Für die römisch-katholische Kirche zusammenfassend: Les archives de l'Église catholique en France, http://www.eglise.catholique.fr/conference-des-eveques-de-france/cef/autres-serviceset-instances/le-centre-national-des-archives-de-leglise-de-france-cnaef/372072-les-archives-deleglise-catholique-en-france/. Für die protestantischen Kirchen (evangelisch, reformiert) siehe die gute Zusammenfassung mit Überblick über die verschiedenen Aufbewahrungsstätten (Kirche, 
öffentliche Archive, Bibliotheken usw.) unter: Guide des Recherches sur l'histoire des familles, Archives cultuelles, http://www.francegenweb.org/ archives/guide/index.php? id=10_cultes_protestants.

26. Dazu ibid., http://www.francegenweb.org/ archives/guide/index.php?id=10_culte_israelite. 27. Vgl. Didier Bondue, Les Archives d'entreprises en France, 2008. Online unter: http:// www.arhivelenationale.ro/images/custom/image/Pdf-uri/Revista/Revista\%202_2008/05\% 20Didier\%20Bondue.pdf.

28. Archives françaises du film, http://www.cnc-aff.fr, vgl. http://fr.wikipedia.org/wiki/ Archives_fran\%C3\%A7aises_du_film.

29. http://www.ina.fr, vgl. http://fr.wikipedia.org/wiki/Institut_national_de_l\%27audiovisuel.

30. Legifrance, Code du patrimoine, http://www.legifrance.gouv.fr/affichCode.do? cidTexte=LEGITEXT000006074236.

31. Archives et archivistes sous le regard de l'historien et La loi sur les archives de 2008 et ses conséquences. Actes de la matinée d'études de la section Archives des administrations centrales de l'Association des archivistes français du 22 novembre 2011 et Actes de la journée d'études du groupe interrégional Bretagne - Pays de la Loire de l'Association des archivistes français du 6 décembre 2011, in: La Gazette des archives 225 (2012-1).

32. Nicht einsehbar sind jedoch Unterlagen, die Aufschluss über Herstellung, Gebrauch und Standorte atomarer, biologischer, chemischer oder vergleichbarer Waffen geben. Die allgemeine Sperrfrist bis zum Ablauf von 30 Jahren nach Entstehung der Unterlagen, wie sie auch die deutschen Archivgesetze vorsehen, wurde 2008 abgeschafft.

33. Archives de France, Accéder aux archives, http://www.archivesdefrance.culture.gouv.fr/ chercher/acces/.

34. Archives de France, Chercher dans les archives, http:// www.archivesdefrance.culture.gouv.fr/chercher/.

35. Archives de France, Guides de recherche, guides et états des sources, http:// www.archivesdefrance.culture.gouv.fr/chercher/organiser/sources/.

36. Christine Nougaret (Hrsg.), Les archives privées: manuel pratique et juridique, Paris 2008 (Manuels et guides pratiques / Direction des Archives de France).

37. Siehe oben Anm. 20; vgl. auch Archivschule Marburg, Archive in Frankreich, http:// archivschule.de/DE/service/archive-im-internet/archive-in-europa/frankreich/archive-infrankreich.html\#fra.

38. Vgl. Archivschule Marburg, Archive in Frankreich, http://archivschule.de/DE/service/ archive-im-internet/archive-in-europa/frankreich/archive-in-frankreich.html\#fra oder den Länderguide Frankreich von Historicum.net, http://www.historicum.net/laender/frankreich/ wissenschaft/archive/.

39. Aus organisatorischen Gründen empfiehlt es sich, solche Anfragen unpersönlich an das Archiv zu richten.

40. Das Nationalarchiv bietet seit Herbst 2013 unter www.archives-nationales.culture.gouv.fr/ siv/ einen virtuellen Repertoriensaal (Salle des inventaires virtuelle) an. Er macht es möglich, von der Konsultation der Findbücher bis zur Bestellung von Dokumenten einen Archivbesuch online vorzubereiten.

41. Lehrmaterial und Übungsbeispiele bieten z. B.: Gabriel Audisio, Isabelle Rambaud, Lire le français d'hier. Manuel de paléographie moderne. XVe-XVIII ${ }^{\mathrm{e}}$ siècles, Paris ${ }^{4} 2012$; Wolfgang Hans Stein, Französisches Verwaltungsschriftgut in Deutschland. Die Departementalverwaltungen in der Zeit der Französischen Revolution und des Empire, Marburg 1996 (Veröffentlichungen der Archivschule Marburg, 24); Theleme - Techniques pour l'Historien en Ligne: Études, Manuels, Exercices, http://theleme.enc.sorbonne.fr/.

42. Vor einer eventuellen Veröffentlichung der Reproduktionen ist eine Rücksprache mit dem Archiv empfehlenswert. 
43. Vgl. etwa Mareike König, Guide Frankreich, in: Clio-online, 27.4.2012, http://www.clioonline.de/guides/frankreich/koenig2012, bes. Nr. 8.

44. http://www.numerique.culture.fr/pub-fr/index.html.

45. Base de données Archim, http://www.culture.gouv.fr/documentation/archim/accueil.html.

46. Etwa: ArchiWebture. Inventaires d'archives d'architectes en ligne, http:// archiwebture.citechaillot.fr/ oder Ministère de la Culture et Communication, Architecture et patrimoine, http://www.culture.gouv.fr/culture/inventai/patrimoine/. Siehe auch oben, Anm. 22 (Catalogue en ligne des archives et des manuscrits de l'enseignement supérieur), Anm. 28 (Archives françaises du film du CNC) und Anm. 29 (Institut national de l'Audiovisuel).

47. Wolfgang Hans Stein (Hrsg.), Inventar von Quellen zur deutschen Geschichte in Pariser Archiven und Bibliotheken, Teil 1, Koblenz 1986; Teil 2: Archive im Bereich des Verteidigungsministeriums, Archive des Außen- und des Finanzministeriums, Stadtpariser Archive und Bibliotheken, Koblenz 2002; Teil 3: Akten zur Französischen Deutschlandpolitik der Zwischenkriegszeit (1918-1940), bearb. von Frank Wittendorfer, 2 Bände, Koblenz 2008 (Veröffentlichungen der Landesarchivverwaltung Rheinland-Pfalz, 39 / 97 / 108).

48. So verwahrt das Pariser Nationalarchiv die Akten der Interalliierten Rheinlandkommission (1918/19-1930) (Elektronisches Inventar unter: http://chan.archivesnationales.culture.gouv.fr/ sdx-23b1-20090531-chan-pleade-2/pl/toc.xsp?

id=FRAN_IR_31006\&fmt=tab\&base=fa\&root=FRAN_IR_31006\&ss=true\&as=true\&ai=HCITR|second| standard|\&idtoc=FRAN_IR_31006-pleadetoc) und die Überlieferung des deutschen Militärbefehlshabers in Frankreich (1940-1944). Im Militärarchiv in Vincennes liegen u.a. die Akten der französischen Rheinarmee, die nach dem Ersten Weltkrieg in Deutschland stationiert war. Die Überlieferung der französischen Besatzungsverwaltung im Deutschland der Jahre nach 1945 befindet sich in einer Außenstelle des Archivs des Quai d'Orsay in Colmar.

49. Deutsches Historisches Institut Paris, http://www.dhi-paris.fr/.

50. Archivum Rhenanum. Blog des Projekts »Grenzüberschreitendes Netzwerk digitaler Geschichtsquellen«, http://archives.hypotheses.org/.

51. Vgl. dazu künftig (voraussichtlich 2014) den Aufsatzband zum 83. Deutschen Archivtag 2013 in Saarbrücken, der das Rahmenthema »Archive ohne Grenzen: Erschließung und Zugang im europäischen und internationalen Kontext« behandelte.

\section{RÉSUMÉS}

Wer anhand historischer Originalüberlieferung forschen möchte, findet in Frankreich eine differenzierte und hochentwickelte Archivlandschaft vor. Während der französische Begriff archives facettenreicher und damit missverständlicher ist als sein deutsches Pendant, fällt die praktische Orientierung in vielen französischen Archiven dank ihrer zentralistischen Einheitlichkeit umso leichter.

\section{INDEX}

Schlüsselwörter : Frankreich, Archivwesen (archivistique), Nationalarchiv (Archives nationales), Archivgesetz (Code du Patrimoine), Findmittel (inventaires), Provenienzprinzip (principe de respect des fonds), Ordnungsschema (cadre de classement) 


\section{AUTEURS}

\section{FLORENCE DE PEYRONNET-DRYDEN}

Florence de Peyronnet-Dryden ist Historikerin und Archivarin.

Mail: info[at]peyronnet-dryden.com

\section{MATTHIAS NUDING}

Dr. Matthias Nuding ist Leiter des Historischen Archivs und des Münzkabinetts im Germanischen Nationalmuseum, Nürnberg.

Mail: m.nuding[at]gnm.de 